University of Wollongong

Research Online

Faculty of Informatics - Papers (Archive)

Faculty of Engineering and Information

Sciences

1977

\title{
A note on asymptotic existence results for orthogonal designs
}

Peter Eades

Jennifer Seberry

University of Wollongong, jennie@uow.edu.au

Nicholas Wormald

Follow this and additional works at: https://ro.uow.edu.au/infopapers

Part of the Physical Sciences and Mathematics Commons

\section{Recommended Citation}

Eades, Peter; Seberry, Jennifer; and Wormald, Nicholas: A note on asymptotic existence results for orthogonal designs 1977.

https://ro.uow.edu.au/infopapers/983

Research Online is the open access institutional repository for the University of Wollongong. For further information contact the UOW Library: research-pubs@uow.edu.au 


\title{
A note on asymptotic existence results for orthogonal designs
}

\author{
Abstract \\ In a recent manuscript "Some asymptotic results for orthogonal designs" Peter Eades showed that for \\ many types of orthogonal designs existence is established once the order is large enough. This paper \\ uses sequences with zero non-periodic and periodic autocorrelation function to establish the asymptotic \\ existence of many orthogonal designs with four variables. Bounds are also established for orthogonal \\ designs of type $(1, k)$ where $k \leq 63$ and $(t)$ where $I \leq 52$. It is shown that any 4 sequences with zero non- \\ periodic auto-correlation function and $8 k-1$ entries +1 or -1 must have length at least $2 k+1$. \\ Disciplines \\ Physical Sciences and Mathematics \\ Publication Details \\ Eades, P, Seberry, J and Wormald, N, A note on asymptotic existence results for orthogonal designs, \\ Combinatorial Mathematics V: Australian Conf. on Combinatorial Mathematics, Melbourne, 1976, 622, in \\ Lecture Notes in Mathematics, Springer--Verlag, Berlin--Heidelberg--New York, 1977, 76-90.
}




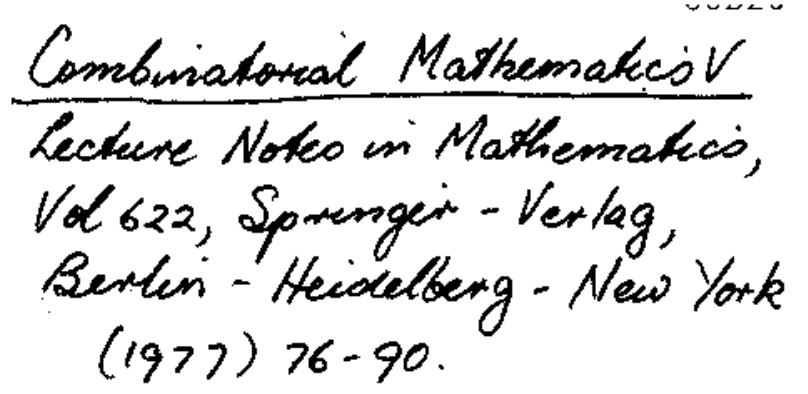

A NOTE ON ASYMPTOTIC EXISTENCE RESULTS FOR

ORTHOGONAL DESIGNS

Peter Eades, Jennifer Seberry Wallis, Nicholas Wormald

In a recent manuscript "Some asymptotic results for orthogonal designs" Peter Eades showed that for many types of orthogonal designs existence is established once the order is large enough.

This paper uses sequences with zero non-periodic and periodic autocorrelation function to establish the asymptotic existence of many orthogonat designs with four variables. Bounds are also established for orthogonat designs of type $(1, k)$ where $k \leq 63$ and ( $\ell$ ) where $\ell \leq 52$.

It is shown that any 4 sequences with zero non-periodic auto-correlation function and $8 k-1$ entries +1 or -1 must have length at least $2 \mathrm{k}+1$.

\section{Introduction}

An orthogonal design of order $n$ and type $\left(u_{1}, u_{2}, \ldots, u_{s}\right)\left(u_{i}>0\right)$ on the commuting variables $x_{1}, x_{2}, \ldots, x_{s}$ is an $n \times n$ thatrix $A$ with entries from $\left\{0, \pm x_{1}, \ldots, \pm x_{s}\right\}$ such that

$$
A A^{t}=\sum_{i=1}^{S}\left(u_{i} x_{i}^{2}\right) I_{n} .
$$

Alternatively, the rows of $A$ are formally orthogonal and each row has precisely $s_{i}$ entries of the type $\pm x_{i}$. 
In [3], where this was first defined and many examples and properties of such designs were investigated, it is mentioned that

$$
A^{t} A=\sum_{i=1}^{s}\left(u_{i} x_{i}^{2}\right) I_{n}
$$

and so the alternative description of A applies equally well to the columns of A. It is also shown in [3] that $s \leq \rho(n)$, where $\rho(n)$ (Radon's function) is defined by

$$
p(n)=8 c+2^{d}
$$

when

$$
\mathrm{n}=2^{\mathrm{a}} \cdot \mathrm{b}, \mathrm{b} \text { odd, } \mathrm{a}=4 \mathrm{c}+\mathrm{d}, 0 \leq \mathrm{d}<4 \text {. }
$$

A weighing matrix of weight $k$ and order $\mathrm{n}$, is a square $\{0,1,-1\}$ matrix, $w=W(n, k)$, of order $n$ satisfying

$$
W W^{t}=k I_{n} .
$$

In [3] it was shown that the existence of an orthogonal design of order $n$ and type $\left(u_{1}, \ldots, u_{s}\right)$ is equivalent to the existence of disjoint weighing matrices $A_{1}, \ldots, A_{s}$, of order $n$, where $A_{i}$ has weight $u_{i}$ and the matrices, $\left\{A_{i}\right\}_{i=1}^{s}$, satisfy the matrix equation

$$
X Y^{T}+Y X^{T}=0
$$

in pairs. In particular, the existence of an orthogonal design of order $\mathrm{n}$ and type $(1, k)$ is equivalent to the existence of a skew-symmetric weighing matrix of weight $k$ anä order $n$.

It is conjectured that:

(i) for $n \equiv 0(\bmod 4)$ there is a weighing matrix of weight $k$ and orden $\mathrm{n}$ for every $\mathrm{k} \leq \mathrm{n}$;

(ii) for $n \equiv 4$ (mod 8) there is a skew-symmetric weighing matrix of order $n$. for every $k<n$, except possibly $k=n-2$, where $k$ is the sum of $\leq$ three squares of integers (equivalently, there is an onthogonal design of type $(1, k)$ in order $n$ for every $k<n$, except possibly. $k=n-2$, which is the sum of $\leq$ three squares of integers).

D. Shapiro and $W$. Wolfe have found powerful algebraic non-existence theorems for orthogonal designs which supercede those of Geramita, Geramita and Wallis [3]. In addition Geramita and Verner [4] and P.J. Robinson [9] have found strong combinatorial theorems. 
Let $R$ be the back diagonal matrix. Then an orthogonal design or weighing matrix is said to be constructed from two circulant matrices $A$ and $B$ if it is of the form

$$
\left[\begin{array}{cc}
A & B R \\
B R & -A
\end{array}\right]
$$

and to be of Goethals-Seidel type if it is of the form

$$
\left[\begin{array}{cccc}
A & B R & C R & D R \\
-B R & A & D^{T} R & -C^{T} R \\
-C R & -D^{T} R & A & B^{T} R \\
-D R & C^{T} R & -B^{T} R & A
\end{array}\right]
$$

where $A, B, C, D$ are circulant matrices.

Hence forth we use $\bar{x}$ for $-x$.

Let $x=\left\{a_{11}, \ldots, a_{1 n}, a_{21}, \ldots, a_{2 n}, \ldots, a_{m l}, \ldots, a_{m n}\right\}$ be $m$ sequences of commuting variables of length $n$.

DEFINITION. (1) The non-periodic auto-correlation function of the fomity of sequences $\mathrm{X}$ (denoted $\mathrm{N}_{\mathrm{X}}$ ) is given by

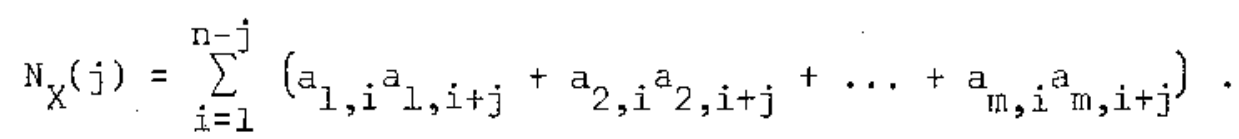

(2) The periodic auto-correlation function of the family of sequences $x$ (denoted $\mathrm{P}_{X}$ ) is given by

$$
P_{X}(j)=\sum_{i=1}^{n}\left(a_{1, i} a_{1, i+j}+q_{2,1} a_{2, i+j}+\ldots+a_{m, i}{ }^{a}, i+j\right)
$$

where we assune the second subscript is actually chosen from the complete set of residues $\bmod n$.

We can interpret the function $\mathrm{P}_{\mathrm{X}}$ in the following way: Form the $\mathrm{m}$ circulant matrices which have first rows respectively,

$$
\left[\begin{array}{llll}
a_{11} & a_{12} & \cdots & a_{1 n}
\end{array}\right],\left[a_{21} a_{22} \cdots a_{2 n}\right], \ldots,\left[a_{m 1} a_{m 2} \ldots a_{m n}\right],
$$

then $P_{X}(j)$ is the sum of the inner products of rows 1 and $j+1$ of these matrices.

Clearly

$$
P_{X}(j)=N_{X}(j)+N_{X}(n-j), j=1, \ldots, n-1,
$$

and 


$$
N_{X}(j)=0 \quad \forall j \Rightarrow F_{X}(j)=0 \quad \forall j .
$$

Note: $P_{X}(j)$ may equal 0 for all $j=1, \ldots, n-1$ even though the $N_{X}^{\prime}(j)$ are not.

Such sequences are used extensively in [5].

THEOREM 3 (Robinson). Alt 4-tuples ( $\mathrm{a}, \mathrm{b}, \mathrm{c}, \mathrm{d}$ ) with $0 \leq \mathrm{a}+\mathrm{b}+\mathrm{c}+\mathrm{d} \leq 32$ are the types of orthogonal designs in order 32 .

THEOREM 4 [3]. AlZ 4-tuples ( $\mathrm{a}, \mathrm{b}, \mathrm{c}, \mathrm{d})$ with $0 \leq a+b+c+d \leq 16$ are the types of orthogonat designs in order 16.

LEMMA 5 [3, 7]. Alt 4-tuples (a,b, c, d) which are not excluded by Wolfe's necessary conditions or the Geramitanerner theorem are

(i) the types of orthogonal designs in order 12 when $\mathrm{a}+\mathrm{b}+\mathrm{c}+\mathrm{d} \leq 12 ;$

(ii) the types of orthogonal designs in order 20 when $a+b+c+d \leq 20$ except possibly for $(1,3,6,8),(1,4,4,9)$ and $(2,2,5,5)$ which are undecided.

the designs may be constructed by using four circulant matrices in the Goethatsseidet array.

\section{Preliminary Results}

LEMMA 6. Suppose there exist circulant matrices $\mathrm{X}_{i}, \mathrm{Y}_{i}, i \in\{1,2, \ldots\}=I$ giving orthogonal designs of type $\left(a_{i}, b_{i}\right)$ in order $2 n$. Then there exists an orthogonat design of type $\left(a_{k}, b_{k}, a_{j}, b_{j}\right), k, j \in I$ in order $4 n$.

COROLLARY 7. There exist orthogonal designs of types $(1,1,13,13)$, $(1,4,9,9),(1,2,4,18),(1,8,8,9),(1,9,9,9),(2,4,4,18),(2,8,9,9)$, $(4,4,9,9)$ and $(5,5,9,9)$ in order 52 .

Proof. First we note there exists $B=w(13,9)$ which is circulant. So there exist $(1,9),(9,9)$ and $(2,18)$ in 26 constructed using two circulant matrices. This fact together with the results of Table 3 give all the designs except $(1,1,13,13)$.

Let $T$ be the circulant matrix which is $l$ above the diagonal and in the (13, I) element and zero elsewhere. Choose $i$ so that the positions of $T^{i}$ and $T^{i+1}$ are zero in $B$. Let $X=T^{i}+T^{i+1}$ and $Y=T\left(q^{2}+q\right) / 2-T\left(q^{2}+q+2\right) / 2$. Then $a B+b X, b B-a X, a Y+c I, b Y+d I$

is the $(1,1,13,13)$ design. 
Designs with zero non-periodic auto-correlation function

$$
\begin{aligned}
& (1,1,1,1) \quad a, b, c, d \\
& (1,1,1,4) \quad a, b, d c \bar{d}, d 0 d \\
& (1,1,2,2) \quad a, b, c d, c d \\
& (1, I, 2,8) \quad \mathrm{d} a \bar{d}, \mathrm{~d} b \bar{d}, \mathrm{~d} c \mathrm{~d}, \mathrm{~d} \bar{c} \mathrm{~d} \\
& (1,1,4,4) \quad b \text { a } \bar{b}, b 0 b, d c \bar{d}, d 0 d \\
& (1,2,2,4) \quad b a \bar{b}, b 0 b, c d, c \bar{d} \\
& (1,2,3,6) \quad a b c, a b \bar{c}, b \bar{a} b, b d \bar{b} \\
& (2,2,2,2) \quad a b, a \bar{b}, c d, c \bar{d} \\
& (1,1,1,9) \text { d a } \bar{d}, d b \bar{d}, d 0 c 0 \bar{d}, d 0 d o d \\
& (1,1,8,8) \quad c d a \bar{d} \bar{c}, \bar{c} d b \bar{d} c, c d 0 d c, c \bar{d} 0 \bar{d} c \\
& (1,1,9,9) \quad b c a \bar{c} \bar{b}, c \bar{b} d b \bar{c}, c c c \bar{c} c, b b b \bar{b} b \\
& (1,4,4,4) \quad b a \bar{b}, b 0 b, c c d \bar{d}, d d \bar{c} c \\
& (2,2,4,4) \quad a b, a \vec{b}, c c d \vec{a}, d d \bar{c} c \\
& (2,3,4,6) \quad \text { a } d \circ \bar{d} a, a d c d \bar{a}, b c \bar{d}, b \bar{c} a \\
& (3,3,3,3) \quad a b c, a \bar{b} 0 d, a 0 c \bar{d}, b c d \\
& (3,3,6,6) \quad a \mathrm{a} b \bar{d} a, a d c d \bar{a}, c \bar{d} a \bar{b}, c \bar{d} \bar{a} 0 b \\
& (4,4,4,4) \quad a b c d, a \bar{b} c \bar{d}, a b \bar{c} \bar{d}, a \bar{b} \bar{c} d \\
& (2,12) \quad a b b \bar{b}, a \bar{b} \bar{b}, b b b, b \vec{b}
\end{aligned}
$$



$(1,1,4,16)$
a 0 a 0 a a , a 0 a b $\vec{a} 0 \bar{a}, a c \bar{a} 0 \bar{a} c a$, a c $\bar{a} d a \bar{c} \bar{a}$
$(1,1,5,5)$
$a, b, c c \bar{c} d 0 d, d d \vec{d} \vec{c} 0 \vec{c}$
$(1,2,4,8)$
$c a \bar{c}, c 0 c, d b \bar{d} 0 d, d b \bar{d} 0 \bar{d}$
$(1,2,6,12)$
$a b 0 b a, a b d \bar{b} \bar{a}, b \bar{a} b b \bar{b}, b \bar{a} b \bar{b} \bar{c}$
$(1,4,5,5)$
$b a \bar{b}, b 0 b, c c \bar{c} 0 d, d d \bar{d} \bar{c} 0 \bar{c}$
$(2,2,2,8)$
$a b, a \bar{b}, c d \bar{c} c 0 c, c d \bar{c} \bar{c} 0 \bar{c}$
$(2,2,4,16)$
a b $\bar{a} a c a, a b \bar{a} a \bar{c} a, a b \bar{a} \bar{a} d \bar{a}, \quad a b \bar{a} \bar{a} \bar{d} \bar{a}$
$(2,2,5,5)$
$a b, a \bar{b}, c c \bar{c} d 0 d, d a \bar{d} \bar{c} 0 \bar{c}$
$(2,2,8,8)$
a b $\bar{a} a 0 a, a b \bar{a} \bar{a} \bar{a}, c d \bar{c} c 0 c, c d \bar{c} \bar{c} 0 \bar{c}$
$(2,2,9,9)$
$\mathrm{a} b \overrightarrow{\mathrm{d}} \mathrm{c} a \bar{c}, \mathrm{~d} b \overline{\mathrm{d}} \overline{\mathrm{c}} \overline{\mathrm{a}} \mathrm{c}, \mathrm{d} c 0 c \overline{\mathrm{c}} \mathrm{c}, \overline{\mathrm{c}} \mathrm{a} 0 \mathrm{~d} c \mathrm{~d}$
$(2,2,10,10)$
$c c \bar{c} d a d, c c \bar{c} d \bar{a} d, d d \bar{d} \bar{c} b \bar{c}, d a \bar{d} \bar{c} \bar{b} \bar{c}$
$(2,4,4,8)$
a b $\bar{a} a \circ a, a b \bar{a} \bar{a}, c_{1} \bar{d} c, \bar{c} d d c$
$(2,4,6,12)$
$a b \bar{c} a b c, a b \bar{c} \bar{a} \bar{c}, b \bar{a} b b c \bar{b}, b \bar{a} b \bar{b} \bar{b}$
$(2,5,5,8)$
a $\mathrm{a} \overline{\mathrm{a}} \mathrm{a} 0 \mathrm{a}, \mathrm{a} \mathrm{d} \overline{\mathrm{a}} \overline{\mathrm{a}} \mathrm{0} \overline{\mathrm{a}}, \mathrm{c} \mathrm{c} \overline{\mathrm{c}} \mathrm{b} 0 \mathrm{~b}, \mathrm{~b} b \overline{\mathrm{b}} \overline{\mathrm{c}} \mathrm{o} \overline{\mathrm{c}}$
$(2,8,8,8)$
$a \mathrm{a} b \bar{b} c d \bar{c}, a a b \bar{b} \vec{c} \bar{d}, a \bar{a} b b c 0 c, a \bar{a} b b \bar{c} 0 \bar{c}$
$(4,4, .4,16)$
$c d \bar{c} c a c b, c d \bar{c} c \bar{a} c \bar{b}, c d \bar{c} \bar{c} a \bar{c} \bar{b}, c d \bar{c} \bar{c} \bar{a} \bar{c} b$
$(4,4,5,5)$
$a \mathrm{ab} \bar{b}, \mathrm{~b} b \overline{\mathrm{a}} \mathrm{a}, \mathrm{c} 0 \mathrm{c} \bar{d} \overline{\mathrm{d}}, \mathrm{d} 0 \mathrm{~d} \overline{\mathrm{c}} \overline{\mathrm{c}} \mathrm{c}$
$(4,4,8,8)$
$a \mathrm{a} b \bar{b} c d, a \mathrm{~d} b \bar{b} \bar{c} \bar{d}, a \bar{a} b b_{c} \bar{d}, a \bar{a} b b \bar{c} d$
$(4,4,10,10)$
$b c a c d d \bar{d}, b \bar{c} a \bar{c} \bar{d} \bar{d} d, b d \bar{a} d \bar{c} \bar{c} c, b \bar{d} \bar{a} \bar{d} c c \bar{c}$
$(5,5,5,5)$
$\mathrm{a} a \overline{\mathrm{a}} \mathrm{b} 0 \mathrm{~b}, \overline{\mathrm{b}} \overline{\mathrm{b}} \mathrm{a} 0 \mathrm{a}, \overline{\mathrm{c}} \overline{\mathrm{c}} \mathrm{d} 0 \overline{\mathrm{d}}, \overline{\mathrm{d}} \overline{\mathrm{d}} \mathrm{d} \mathrm{c} 0 \mathrm{c}$
$(6,6,6,6)$
$a \mathrm{a} b \bar{b} c d, \bar{b} \bar{b} a \bar{a} a \bar{c}, \bar{c} \bar{c} \bar{d} a \mathrm{ab}, \overline{\mathrm{d}} \overrightarrow{\mathrm{d}} \mathrm{c} \bar{c} \bar{b} a$
$(7,7,7,7)$
$a \mathrm{a} \overline{\mathrm{a}} \mathrm{b} c \mathrm{~b} d, \overline{\mathrm{b}} \overline{\mathrm{b}} \mathrm{a} a \mathrm{~d} \overline{\mathrm{c}}, \overline{\mathrm{c}} \overline{\mathrm{c}} \mathrm{c} \overline{\mathrm{d}} \mathrm{a} \overline{\mathrm{d}} \mathrm{b}, \overline{\mathrm{d}} \overline{\mathrm{d}} \mathrm{d} c \bar{b} \mathrm{c} a$ 
LEMMA 8. There exists $a(1,1,2,4,9)$ design in 24 and $(1,1,1,4,20)$ and $(1,1,9,8,8)$ designs in 40 .

Proof. Use

$$
\begin{aligned}
& \mathrm{abb}, \overrightarrow{\mathrm{abb}}, 0 \mathrm{cc}, 0 \mathrm{~b} \overline{\mathrm{b}}, \mathrm{d} 00, e 00, \overline{\mathrm{bbb}}, 0 \mathrm{c} \overline{\mathrm{c}}
\end{aligned}
$$

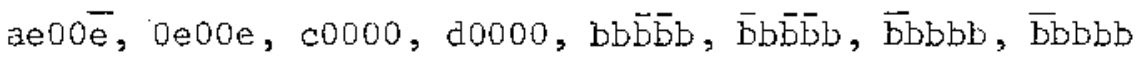

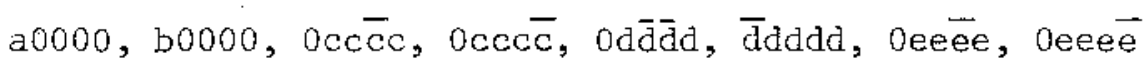

as the first rows of circulant matrices in Lemna 5 parts (viii), (iii) and (viii) respectively of $[11]$.

\section{Main Results}

THEOREM 9. Sequences of comuting variables with zero nonmeriodic autocorrelation function exist which give the following orthogonal designs in order $4 \mathrm{n}$, $n \geq 7:$

$$
\begin{array}{lllll}
(1,1,1,1) & (1,2,2,4) & (2,2,5,5) & (3,3,6,6) \\
(1,1,1,4) & (1,2,3,6) & (2,2,8,8) & (4,4,4,4) \\
(1,1,1,9) & (1,2,4,8) & (2,2,9,9) & (4,4,4,16) \\
(1,1,2,2) & (1,2,6,12) & (2,2,10,10) & (4,4,5,5) \\
(1,1,2,8) & (1,4,4,4) & (2,3,4,6) & (4,4,8,8) \\
(1,1,4,4) & (1,4,5,5) & (2,4,4,8) & (4,4,10,10) \\
(1,1,4,16) & (2,2,2,2) & (2,4,6,12) & (5,5,5,5) \\
(1,1,5,5) & (2,2,2,8) & (2,5,5,8) & (6,6,6,6) \\
(1,1,8,8) & (2,2,4,4) & (2,8,8,8) & (7,7,7,7) . \\
(1,1,9,9) & (2,2,4,16) & (3,3,3,3) &
\end{array}
$$

Proof. Table 1 has the sequences which should be used as first rows of circulant matrices in the Goethals-Seidel array to obtain the result.

LEMMA 10. The following orthogonat designs in order $4 \mathrm{n}$ can be constructed by using sequences of commuting variables with zero non-pexiodic auto-corretation function to form circulant matrices which are then used in the Goethals-seidel array:

$$
\begin{aligned}
& \text { (i) }(1,2,3,6),(1,1,4,4),(1,1,2,8) \text { for } n \geq 3 ; \\
& \text { (ii) }(1,1,8,8),(1,1,9,9),(1,4,4,4) \text { for } \mathrm{n} \geq 5 ; \\
& \text { (iii) }(1,1,4,16),(1,2,6,12) \text { for } \mathrm{n} \geq 6 ; \\
& \text { (iv) }(1,24),(23),(26),(1,27) \text { for } \mathrm{n} \geq 7 ; \\
& \text { (v) }(29),(30) \text { for } \mathrm{n} \geq 8 ; \\
& \text { (vi) }(1,1,32),(1,35),(31) \text { for } \mathrm{n} \geq 9 ; \\
& \text { (vii) }(1,36),(38) \text { for } \mathrm{n} \geq 10 ; \\
& \text { (viii) (1, 43), (1, 2, 2, 36), (39), (42) for } \mathrm{n} \geq 11 ;
\end{aligned}
$$




\section{Table 2.}

Designs with zero non-periodic auto-correlation function

$(1,1,32)$

$(1,33)$

$(1,35)$

$(1,36)$

(38)

$(1,2,2,36)$

$(1,43)$

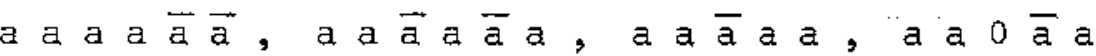
a $\bar{a}$ a a a $\bar{a}$, a a $\bar{a} \bar{a} \bar{a}$ a $\bar{a}$, a $\bar{a} \bar{a} 0 \bar{a} \bar{a}$, a 0 a 000 a $a b b \bar{b} b \bar{b} \bar{b}, \bar{a} b \bar{b} b \bar{b} \bar{b}, b b b \bar{b} b b, b \bar{b} \bar{b} \bar{b} \bar{b}$ a a $\bar{a} b a \bar{a} \bar{a}$, a a $0 \bar{a} \bar{a}$, a a $\bar{a} a \bar{a} \bar{a}$, a a a a $\bar{a} a$

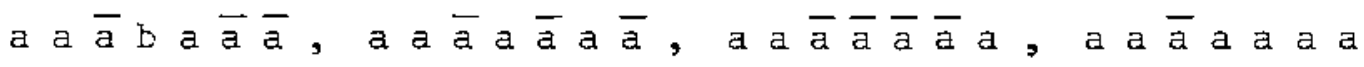

a a a $\bar{a} \bar{a}$ a $\bar{a}$, a a a $\overline{\mathrm{a}}$ a $\overline{\mathrm{a}} \overline{\mathrm{a}} \mathrm{a}$, a a a $\overline{\mathrm{a}} \mathrm{a} \overline{\mathrm{a}} \mathrm{a}$, a a a $0 \overline{\mathrm{a}} \mathrm{a}$ a a a a $\vec{a} a$ a $\vec{a} \bar{a}, \quad$ a a a $a \bar{a} a \bar{a} a$, a a $\bar{a} \bar{a} a \bar{a} a$, a a a a $\bar{a} \bar{a}$

a a $\bar{a}$ a a a $\bar{a}$ a $\bar{a}$, a a $\bar{a}$ a $0 \bar{a}$ a $\bar{a} a$, a a a a a $\bar{a} \bar{a}$, a a a $\bar{a} \bar{a}$ a a

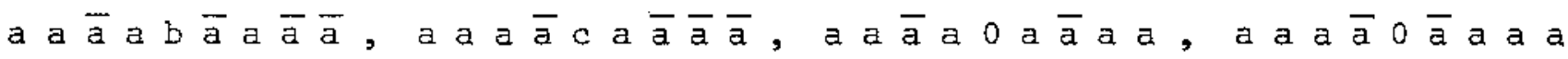
a a a $\bar{a} \mathrm{~b}$ a $\overline{\mathrm{a}} \overline{\mathrm{a}} \overline{\mathrm{a}}$, a a a $\overline{\mathrm{a}} \overline{\mathrm{a}} \mathrm{a} \overline{\mathrm{a}} \mathrm{a}, \mathrm{a}$, a $\overline{\mathrm{a}} \mathrm{a} \overline{\mathrm{a}} \mathrm{a}$, a a a $\overline{\mathrm{a}} \mathrm{a} \overline{\mathrm{a}} \mathrm{a} \mathrm{a}$

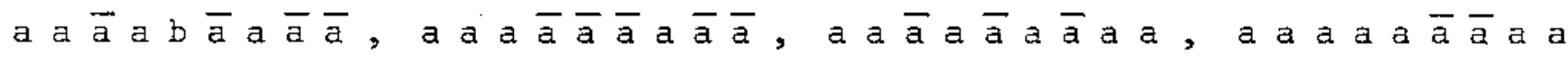

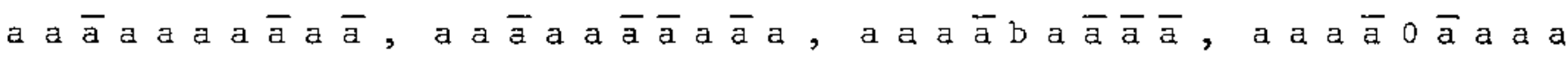

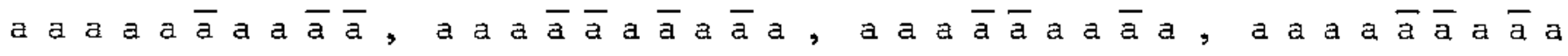

a a a $\bar{a}$ a $\bar{a}$ a a $\bar{a} \bar{a} \bar{a}$, a a a $\bar{a}$ a 0 a a $\bar{a} a$, a a a $\bar{a} \bar{a}$ a $\bar{a} \bar{a} a$, a a $\bar{a}$ a $\bar{a} \bar{a} \bar{a}$

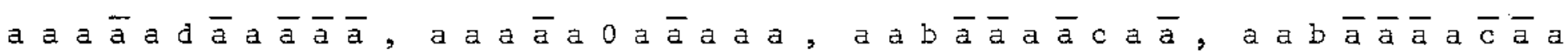

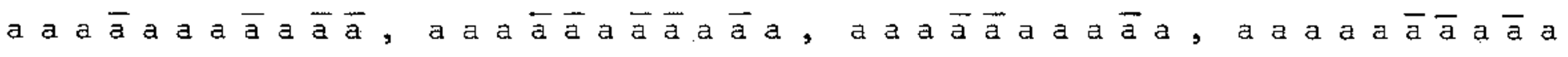

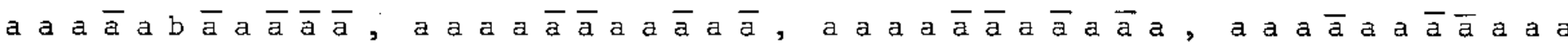


$(1,44)$

$(1,1,40)$

(47)

(48)

(50)

(1, 51)

$(1,1,17,17)$

$(1,2,66)$ a a $\bar{a} \bar{a}$ a $\bar{a}$ a $\bar{a} \bar{a} \bar{a}$ a, a a $\vec{a} \bar{a}$ a $\bar{a} \bar{a} a$ a a a $\bar{a}$, a $\bar{a}$ a a a b $\bar{a} \bar{a} \bar{a} \bar{a}$, a $\bar{a}$ a a a 0 a a a $\bar{a}$ a



a 0 a $\bar{a} \bar{a}$ a 0 a $\bar{a} \bar{a} 0$ a

a a a $\bar{a}$ a a $\bar{a}$ a a $\bar{a} \bar{a} \vec{a}$, a a a $\vec{a}$ a $\bar{a} 0 \bar{a}$ a $\bar{a} \bar{a} a a$, a a a $\bar{a}$ a a a a $\bar{a} \bar{a}$,

a a a $\bar{a}$ a $\bar{a} \bar{a}$ a $\bar{a} \bar{a}$

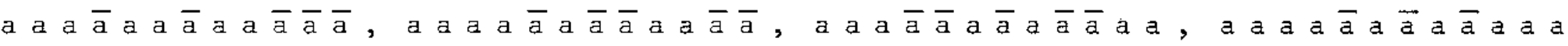
a a a a $\bar{a}$ a $\bar{a}$ a a $\bar{a} \bar{a} \bar{a}$, a a a a $\bar{a}$ a $\bar{a} \bar{a}$ a a $\bar{a} \bar{a}$ a, a a a $\bar{a} \bar{a} a \bar{a} a \bar{a} \bar{a} a \bar{a}$,

a a a a a $\bar{a}$ a $\bar{a}$ a $\bar{a}$ a $a$

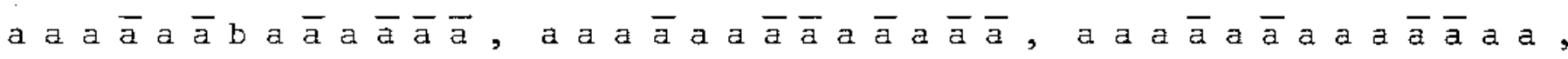

a a a a a $\bar{a} \bar{a}$ a $\bar{a} \bar{a} a$ a

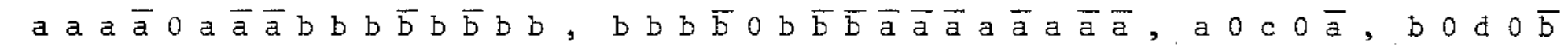
a a a $\mathrm{a} b$ a $\bar{a} \bar{a} \bar{a} a$ a a $\bar{a} \bar{a} a \bar{a} \bar{a} a$, a a a $\bar{a} b$ a $\bar{a} \bar{a} \bar{a} \bar{a} \bar{a} \bar{a} a \bar{a} a \bar{a}$,

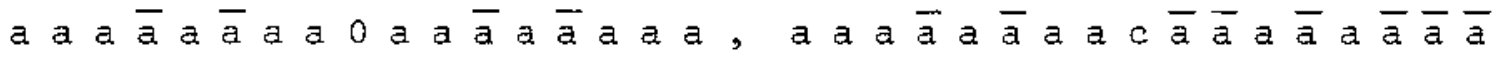




$$
\begin{aligned}
& \text { (ix) }(1,44),(48) \text { for } \mathrm{n} \geq 12 ; \\
& \text { (x) }(47),(50),(1,51) \text { for } \mathrm{n} \geq 13 ; \\
& \text { (xi) }(1,1,17,17) \text { for } \mathrm{n} \geq 16 ; \\
& \text { (xii) }(1,2,66) \text { for } \mathrm{n} \geq 18 ; \\
& \text { (xiii) }(10,10,13,13) \text { for } \mathrm{n} \geq 14 . \\
& \text { Proof. Use the sequences from Tables } 1 \text { and } 2 \text { for }(i)-(x) \text {. For }(x i i i) \text { use the }
\end{aligned}
$$
appropriate sequences of Table 3 .

COROLLARY 11. There exist weighing matrices $\mathrm{W}(\mathrm{n}, \mathrm{k})$ for $\mathrm{k}=0,1, \ldots, \mathrm{n}$ when $\mathrm{n}=12,20,28,36,44$ constmuted using four circulant matrices in the Goethals-Seidel array EQUIVALENTLY the weighing matrix conjecture is true for $n=12,20,28,36,44$.

Proof. Use Tables 1 and 2.

THEOREM 12. There exist orthogonal designs of order $4 \mathrm{n}$ and type $(1, k)$ when

$$
\text { (i) for } n \geq t, t=3,5,7, k \in\left\{x: x \leq 4 t-1, x=a^{2}+b^{2}+c^{2}\right\} \text {; }
$$




\section{Table 3.}

Sequences with zero non-periodic auto-correlation function

\begin{tabular}{|c|c|c|}
\hline Length & Type & \\
\hline 1 & $(1,1)$ & $a, b$ \\
\hline 2 & $(2,2)$ & $a b, a \bar{b}$ \\
\hline 3 & $(1,4)$ & $b a \vec{b}, b 0 b$ \\
\hline 4 & $(4,4)$ & $a \mathrm{a} b \bar{b}, \quad b \quad b \bar{a} a$ \\
\hline 6 & $(2,8)$ & $\mathrm{a} b \bar{a} a 0 a, a b \bar{a} \bar{a} 0 \bar{a}$ \\
\hline 6 & $(5,5)$ & $a \mathrm{a} \bar{a} \mathrm{~b} 0 \mathrm{~b}, \mathrm{~b} b \overline{\mathrm{b}} \overline{\mathrm{a}} 0 \overline{\mathrm{a}}$ \\
\hline 8 & $(8,8)$ & $\mathrm{a} a \mathrm{a} \overline{\mathrm{a}} \mathrm{b} b \overline{\mathrm{b}} \mathrm{b}, \overline{\mathrm{b}} \overline{\mathrm{b}} \overrightarrow{\mathrm{b}} \mathrm{b} a \mathrm{a} \overline{\mathrm{a}} \mathrm{a}$ \\
\hline 10 & $(4,16)$ & $a b \bar{a} a \bar{a} a \bar{a} \bar{a} b a, a b \bar{a} a a a$ a $a \bar{b} \bar{a}$ \\
\hline I0 & $(10,10)$ & $a \bar{a} \bar{a} a b b b b \bar{b}, b \bar{b} \bar{b} b \bar{a} b \bar{a} \bar{a} \bar{a} a$ \\
\hline 11 & $(13)$ & a a a $0 \bar{a}$ a $a 0 \vec{a} a \vec{a}$, a 0 a $000 \bar{a} 000$ \\
\hline 14 & $(13,13)$ & $a \mathrm{a} a b \bar{a} a \mathrm{a} \bar{b} \bar{a} a \bar{a} b \circ b, b b \quad \bar{a} \bar{b} b b a \bar{b} b \bar{b} \bar{a} 0 \bar{a}$ \\
\hline
\end{tabular}




\section{Table 4.}

Sequences with zero periodic auto-correlation function
$n \geq 5 \quad(1,14)$
a $0_{\frac{1}{2}(n-5)} \mathrm{b} b \bar{b} \bar{b} 0_{\frac{3}{2}(n-5)}, \quad b b \bar{b} b 0_{n-4}, b b b o_{n-3}, b \bar{b} 0_{n-3}$
$\mathrm{n} \geq 7$
$(1,1,1,16)$
a $o_{\frac{1}{2}(n-7)}$ b b Б b b $\bar{b} o_{\frac{1}{2}(n-7)}, c o_{\frac{3}{2}(n-5)}$ b $00 \bar{b} \cdot o_{\frac{1}{2}(n-5)}$,
d $o_{\frac{1}{2}(n-5)}$ b $00 \bar{b} O_{\frac{1}{2}(n-5)}, \quad$ b b $\bar{b}$ b b b $0_{n-7}$
$(1,1,13,13)$
b $o_{\frac{7}{2}(n-7)} \subset \subset \bar{c} c \bar{c} \bar{c} o_{\frac{1}{2}(n-7)}, \quad c c d \bar{c} d c d o_{n-7}$, d d $\bar{c} \bar{d} \bar{c} \bar{c} o_{n-7}$,
a $O_{\frac{z}{2}(n-7)} d \mathrm{~d} \overline{\mathrm{d}} \mathrm{d} \overline{\mathrm{d}} \overline{\mathrm{d}} \hat{O}_{\frac{t}{2}(n-7)}$
$(1,1,26)$



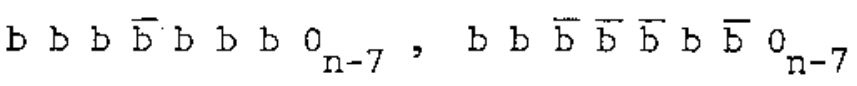
$(1,22)$
a $0_{\frac{3}{2}(n-7)} \mathrm{b} b \mathrm{~b} \overline{\mathrm{b}} \overline{\mathrm{b}} 0_{\frac{z_{2}(n-7)}{}}, \mathrm{b} \mathrm{b} \overline{\mathrm{b}} \mathrm{b} \overline{\mathrm{b}} \mathrm{b} 0_{n-6}, \mathrm{~b} \mathrm{~b} \overline{\mathrm{b}} \mathrm{b} \mathrm{o}_{n-5}$,
b
$\mathrm{n} \geq 9 \quad(1,25)$

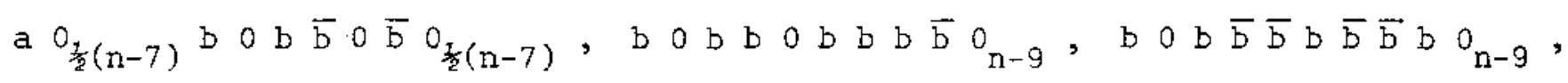
$\mathrm{b} b \overrightarrow{\mathrm{b}} \overrightarrow{\mathrm{b}} \overline{\mathrm{b}} \mathrm{b} \quad \mathrm{O}_{\mathrm{n}-6}$

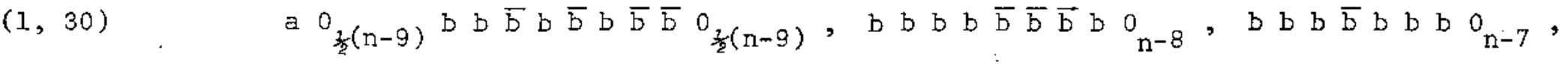
$\mathrm{b} \overline{\mathrm{b}} \overline{\mathrm{b}} \mathrm{b} \overline{\mathrm{b}} \overline{\mathrm{b}} \mathrm{b} \quad \mathrm{o}_{\mathrm{n}-7}$ 


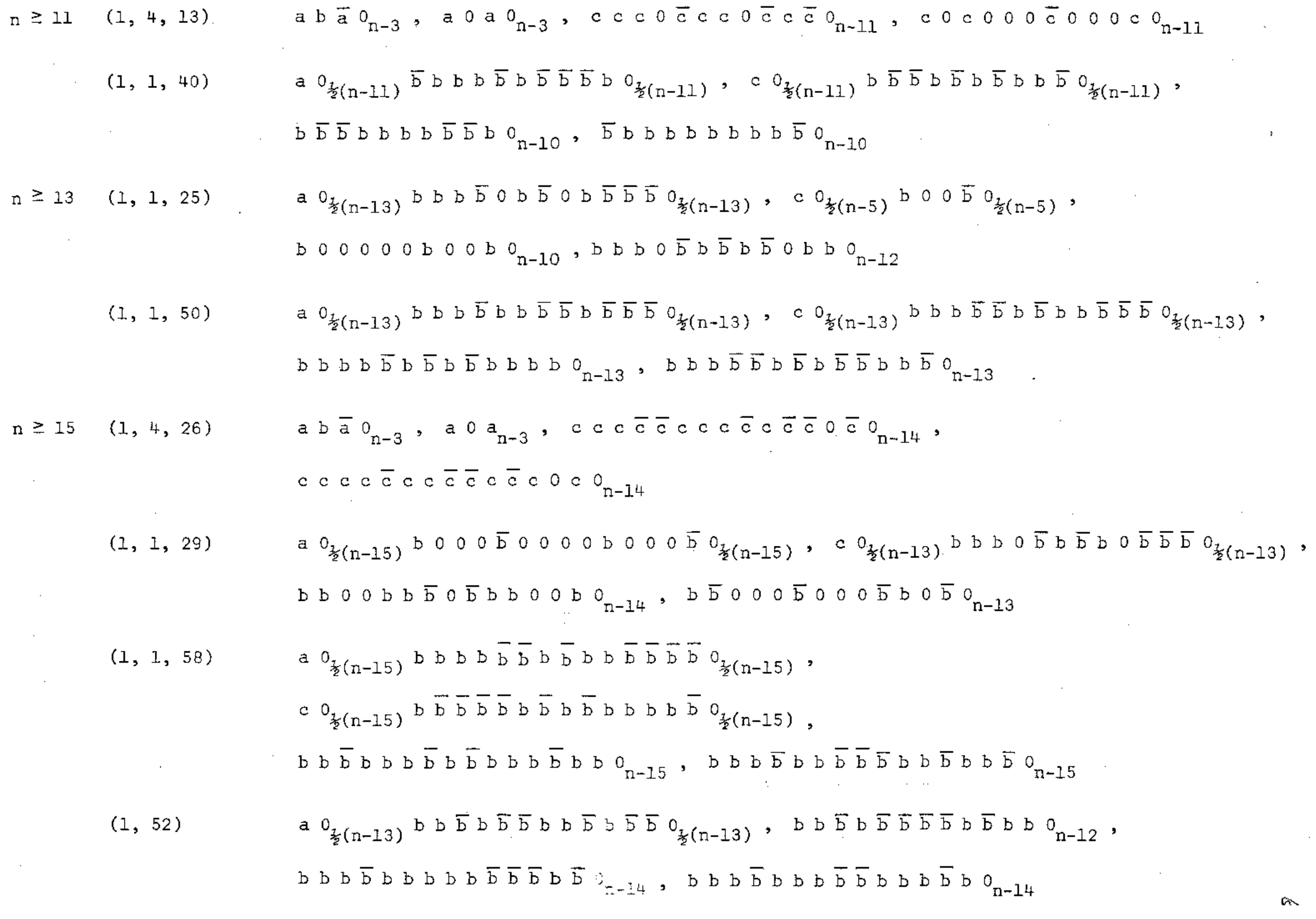


(ii) for $n \geq 9, k \in\left\{x: x \leq 35, x=a^{2}+b^{2}+c^{2}, x \neq 34\right\}$;

(iii) for $n \geq 11, k \in\left\{x: x \leq 43, x=a^{2}+b^{2}+c^{2}, x \neq 34,37,42\right\}$;

(iv) for $n \geq 13, k \in\left\{x: x \leq 51, x=a^{2}+b^{2}+c^{2}, x \neq 34,37,42,45,46\right.$, $48,49\}$;

(v) for $n \geq 15, k \in\left\{x: x \leq 59, x=a^{2}+b^{2}+c^{2}, x \neq 34,37,42,45,46\right.$, $48,49,53,54,56,57\} ;$

(vi) for $\mathrm{n} \geq 16, \mathrm{k} \in\left\{\mathrm{x}: \mathrm{x} \leq 63, \mathrm{x}=\mathrm{a}^{2}+\mathrm{b}^{2}+\mathrm{c}^{2}, \mathrm{x} \neq 37,42,45,46,48\right.$, $49,53,54,56,57,60,61,62,63\}$.

In all cases when $\mathrm{n}$ is odd it is possible to construct the designs using four circulant matrices in the Goethals-seidel array.

Proof. We use Table 1 to obtain the result for $n \geq 3$ and $k=12,16,17,18,19$ for $n \geq 5$. Table 4 gives $(1,14)$ for odd $n \geq 5$ and $(1,13)$ for odd $n \geq 7$. We noted above that $(1,23)$ exists in 20 and both $(1,13)$ and $(1,14)$ exist in 16 and 24 which gives the result.

For $n \geq 7$ Tables 2 and 4 give the result immediately except for $(1,25)$. Table 4 gives $(1,25)$ for $n \geq 9$ and its existence in $32,40,48$, 56 follows because $(1,1,12,12)$ exists in these orders. The existence of $(1,25)$ in order 28 gives the result for $(1,25)$.

Tables 1,2 and 4 give the result immediately except for $(1,29)$ and (1, 30). Table 4 gives these two in odd orders $\geq 15$.

Table 2 gives the result immediately for $(1,32)$ and $(1,33)$ in orders $\mathrm{n} \geq 9$. Table 4 gives $(1,30)$ for $\mathrm{n}$ (odd) $\geq 9$ and $(1,29)$ for $\mathrm{n}$ (odd) $\geq 15$. Both $(1,30)$ and $(1,29)$ exist in onders $32,40,48$ and 56 . We exhibit first rows of four circulant matrices which give $(1,29)$ in onders 36,44 and 52 :

$36: a b b \bar{b} b \bar{b} \bar{b} \bar{b}, 0 b b b \bar{b} \bar{b} \bar{b} \bar{b}, b \bar{b} 0 b b b b 0 b, b \bar{b} 0 b 0 b 0 \bar{b}$ 44: a b $\bar{b} b \mathrm{~b} \bar{b} \bar{b} \bar{b} \bar{b}, \bar{b} b 0 b b b 000 b 0, b b b 0 \bar{b} b 0 \bar{b} \bar{b}$, b 0 b

52: $\quad \mathrm{b} 0 \mathrm{blO}_{10}, \mathrm{~b} a \overline{\mathrm{b}} \mathrm{O}_{10}, 0 \mathrm{~b} \overrightarrow{\mathrm{b}} \overline{\mathrm{b}} \overline{\mathrm{b}} \overline{\mathrm{b}} \overline{\mathrm{b}} \mathrm{b} \overline{\mathrm{b}} \overrightarrow{\mathrm{b}} \mathrm{b}$,

b $\bar{b} b \bar{b} \bar{b} \bar{b} \bar{b} \bar{b} \bar{b}$.

This gives the result.

For $n \geq 11$ we consider $k \in\{36,38,40,41,43\}$. Tables 2,4 and the existence of $(1,41)$ in order 48 gives the result.

For $n \geq 13$ we consider $k \in\{44,50,51\}$. Table 2 gives the result for $k=44,51$. Table 4 gives $(1,50)$ for $\mathrm{n}$ (odd) $\geq 13$ so the existence of $(1,50)$ in $56,64,72, \ldots$ (which follows since $(1,25)$ exists in $4 m, m \geq 7$ ), gives 
the result.

Table 4 gives $(1, k)$ for $k \in\{52,58,59\}$ in orders $4 n, n$ (odd) $\geq 15$. $(1,26)$ and $(1,29)$ exist in orders 32 and $4 m, m \geq 9$. Hence we have $(1,52),(1,58)$ and $(1,59)$ in orders $64,72,80, \ldots$ which gives the result. Table 2 gives $(1,34)$ in all $4 m, m \geq 16$.

\section{Comments on the Tables}

Table 2 was constructed using the Research School of Physical Sciences, DEC-10 System. The results obtained led by various devious methods to Tables 1 and 4.

We now discuss the analysis and method which led to the results of Table 2 but first a definition:

DEFINITION. The weight of m-complementary sequences of length $\mathrm{n}$ is thein total number of non-zero entries.

$$
\text { We consider 4-complementary sequences } A=\left\{A_{1}, A_{2}, A_{3}, A_{4}\right\} \text { where }
$$
$A_{i}=\left\{a_{i, 1}, a_{i, 2}, \ldots, a_{i, n}\right\}, i=1,2,3,4$, of length $n$ with entries $1,-1$ or 0 ; we write $s_{i j}=N_{A_{i}}(j)(\bmod 2)$. Since the sequences $A$ are complementary

$$
N_{\Lambda}(j)=\sum_{i=1}^{4} N_{A_{i}}(j)=0 \quad \forall j=1, \ldots, n-1 .
$$

Hencè

$$
\sum_{i=1}^{4} s_{i j} \equiv 0(\bmod 2) . \quad \forall j=1, \ldots, n-1
$$

and thus

$$
\sum_{j=1}^{n-1} \sum_{i=1}^{4} s_{i j}=\sum_{i=1}^{4} \sum_{j=1}^{n-1} s_{i j} \equiv 0(\bmod 2) .
$$

We use this to prove

LEMMA 13. Any 4-complementary sequences of weight $8 \mathrm{k}-1$ have length at Least $2 k+1$.

Proof. It is sufficient to show this for sequences of one variable, so suppose $A_{i}$ as above have weight $8 k-1$. Further suppose, contrary to the lemma, that the length $\mathrm{n}$ is $2 \mathrm{k}$. Then there is precisely one zero anongst the four sequences assume it is in $A_{1}$. It follows that

$$
s_{2 j} \equiv s_{3 j} \equiv s_{4 j} \equiv n-j(\bmod 2)
$$

and so using $n=2 k$, 


$$
\sum_{i=2}^{4} \sum_{j=1}^{n-1} s_{i j} \equiv k(\bmod 2) .
$$

Now the single zero element of $A_{l}$ must occur in precisely, $n-1=2 k-1$ of the pairs $\left\{\left(a_{1, x}, a_{1, y}\right\}, x \neq y\right\}$, and thus

$$
\begin{aligned}
\sum_{j=1}^{n-1} s_{1 j} & \equiv \frac{n(n-I)}{2}-(2 k-1) \\
& \equiv k+I(\bmod 2) \quad \text { (using. } n=2 k \text { ) }
\end{aligned}
$$

which contradicts (2) when ( 3 ) is considered.

The lemma now follows, since the length of the four sequences must be at least $2 \mathrm{k}$ if their weight is $8 \mathrm{k}-1$.

A computer search has been made for 4-complementary sequences with one variable, of various weights $w$. Whenever $w \neq 7$ (mod 8$)$, the seanch has been successful for sequences of length $\left[\frac{w+3}{4}\right]$ before exhausting all possibilities (square brackets denoting integer part as usual); when $w=8 k+7$ this is true of sequences of length $2 k+1$, which is the best possible considering the above lemma.

The search for the sequences by computer is simplified if the positions of zeros are considered before hand. Note that these positions completely deternine $s_{i j}$ for each $i$ and $j$. If sequences of length $n$ and weight $w$ are required, the number of zeros is known, and in general there are not many configurations of positions of zeros which will satisfy equation ( 1 ). For example, if $w=8 k+3$ and $n=2 k+1$, the only zero must be the $(k+1)$ th entry of one of the sequences.

In fact, no case has been found of a configuration of zeros which has resultant $s_{i j}$ satisfying $(1)$, but for which there are no complementary sequences.

Once the desired configuration of zeros was deternined, the search was made in a straight-forward manner by taking first the non-zero elements amongst

$$
T_{1}=\left\{a_{1,1}, a_{2,1}, a_{3,1}, a_{4,1}, a_{1, n}, a_{2, n}, a_{3, n}, a_{4, n}\right\},
$$

progressing through all possible combinations until one was found satisfying $x_{n-1}=0$, thence proceeding to non-zero elements amongst

$$
T_{2}=\left\{a_{1,2}, a_{2,2}, a_{3,2}, a_{4,2}, a_{1, n-1}, a_{2, n-1}, a_{3, n-1}, a_{4, n-1}\right\}
$$

and finding a combination satisfying $x_{n-2}=0$, etc. (Exhaustion of ali possibilities for $T_{i}$ of course eliminates the current values for $\left.T_{i-I}.\right)$ In this manner, after suitable values for $T_{n / 2}$ were found, or, if $n$ is odd, for 
$\left.\mathrm{T}_{(\mathrm{n}+1) / 2}=\left\{\mathrm{a}_{1,(\mathrm{n}+1) / 2}, \mathrm{a}_{2,(\mathrm{n}+1) / 2}, \mathrm{a}_{3,(\mathrm{n}+1) / 2}, \mathrm{a}_{4,(\mathrm{n}+1) / 2}\right\}\right)$ the remainder of the conditions $x_{j}=0$ were checked. In this way an exhaustive search, for a given configuration of zeros, was carried out.

\section{References}

[1] Peter Eades, "Some asymptotic existence results for orthogonal designs", Ars. combinatoria.

[2] Peter. Eades and Jennifer Seberry Wallis, "Some asymptotic results for orthogonal designs: II", Colloque sur Problèmes Combinatoires et Theorie des Graphes, Orsay, France, 1976.

[3] Anthony V. Ceramita, Joan Murphy Geramita, Jennifer Seberry Wallis, "Orthogonal designs", Linear and Multilinear Algebra

[4] A.V. Geramita, J.H. Verner, "Onthogonal designs with zero diagonal", Conad. J. Math.

[5] Anthony V. Geramita, Jennifer Seberry Wallis, "Orthogonal designs II", Aequationes Math. 13 (1975), 299-313.

[6] Anthony V. Geramita, Jennifer Seberry Wallis, "Orthogonal designs III: weighing matrices", Utilitas Math. 6 (1974), 209-236.

[7]' Anthony V. Geramita, Jennifer Seberry Wallis, "Orthogonal designs IV: existence questions", J. Combinatorial Theory Ser. A 19 (1975), 66-83.

[8] Marshall Hall, Jr., Combinatorial Theom, Blaisdell, Waltham Mass., 1967.

[9] Peter J. Robinson, Private Communication, 1976.

[10] D. Shapiro, Private Communication, 1975.

[11] Jennifer Seberry Wallis, "Orthogonal designs $V$ : orders divisible by eight", Utilitas Math. 9 (1976), 263-281.

[12] Warren W. Wolfe, "Rational quadratic forms and orthogonal designs", J. Number Theory.

Department of Pure Mathematics, School of General Studies, Australian National University, Canberra, ACT, Australia;
Department of Mathematics, Institute of Advanced Studies, Australian National University, Canberra, ACT, Australia;

Department of Mathematics, University of Newcastle, New South Wales, Australia. 